\title{
Correction to: Guiding the Training of Distributed Text Representation with Supervised Weighting Scheme for Sentiment Analysis
}

\author{
Zhe Zhao ${ }^{1}$ Tao $\mathrm{Liu}^{1} \cdot$ Shen $\mathrm{Li}^{2} \cdot$ Bofang $\mathrm{Li}^{1} \cdot$ Xiaoyong $\mathrm{Du}^{1}$
}

Published online: 1 December 2017

(c) The author(s) 2017. This article is an open access publication

\section{Correction to: Data Sci. Eng. (2017) 2:178-186 \\ https://doi.org/10.1007/s41019-017-0040-6}

In the originally published article, the acknowledgment section is missing. Please find it as follows.

Acknowledgements This work is supported by the Fundamental Research Funds for the Central Universities, and the Research Funds of Renmin University of China, the Outstanding Innovative Talents Cultivation Funded Programs 2016 of Renmin University of China. This work is partially supported by National Natural Science Foundation of China with Grant No. 61472428, ECNU-RUC-InfoSys Joint Data Science Lab and a gift from Tencent.
Open Access This article is distributed under the terms of the Creative Commons Attribution 4.0 International License (http://creative commons.org/licenses/by/4.0/), which permits unrestricted use, distribution, and reproduction in any medium, provided you give appropriate credit to the original author(s) and the source, provide a link to the Creative Commons license, and indicate if changes were made.
The original article can be found online at https:// doi.org/10.1007/s41019-017-0040-6.

Tao Liu

tliu@ ruc.edu.cn

Zhe Zhao

helloworld@ruc.edu.cn

1 School of Information, Renmin University of China, Information building, Beijing, China

2 Institute of Chinese Information Processing, Beijing Normal University, Beijing, China 